\title{
Tackling antimicrobial resistance by exploring new mechanisms of antibiotic action
}

\author{
Cruz L Matos de Opitz ${ }^{1}$ (D) \& Peter Sass*,1,2 (iD \\ ${ }^{1}$ Department of Microbial Bioactive Compounds, Interfaculty Institute of Microbiology \& Infection Medicine, University of \\ Tübingen, Auf der Morgenstelle 28, 72076 Tübingen, Germany \\ ${ }^{2}$ German Center for Infection Research (DZIF), Partner Site Tübingen, Germany \\ *Author for correspondence: peter.sass@uni-tuebingen.de
}

\begin{abstract}
"Mainly due to the intensive use and/or misuse of antibiotics in agriculture and health sectors, we are facing the emergence and spread of bacteria that are resistant to one, several or even all clinically applied antibiotic classes."
\end{abstract}

First draft submitted: 21 February 2020; Accepted for publication: 15 May 2020; Published online: 10 July 2020

Keywords: ADEP • afabicin $\bullet \beta$-lactones $\bullet$ brilacidin $\bullet$ cyclomarin A $\bullet$ drug discovery $\bullet$ murepavadin $\bullet$ ribocil $\bullet$ ridinilazole $\bullet$ teixobactin

\section{Antimicrobial resistance: a global challenge}

Antibiotics are undoubtedly one the most influential discoveries in medicine. Following their introduction in the first half of the last century, the steadily increasing use of antibiotics as therapeutic agents has permitted the effective treatment of even complicated, life-threatening bacterial infections, for example, septicemia and tuberculosis, thereby saving millions of lives. Moreover, antibiotics have become essential instruments in modern medicine, facilitating a wide range of medical procedures such as transplantation, orthopedic surgery and cancer chemotherapy. Hence, antibiotics have expanded the available options in the invasive medical field, thereby increasing the quality of human life and decreasing morbidity and mortality related to bacterial infections.

Today, antimicrobial resistance (AMR) has become prevalent and multidrug-resistant (MDR) pathogens are seriously challenging our community and healthcare systems. Mainly due to the intensive use and/or misuse of antibiotics in agriculture and health sectors, we are facing the emergence and spread of bacteria that are resistant to one, several or even all clinically applied antibiotic classes. As a consequence, serious bacterial infections are becoming harder to treat or even untreatable. The World Health Organization (WHO) acknowledges AMR as one of the most severe threats to human and animal health [1]. Also, the scenario is not expected to significantly improve in the near future, since only few innovative antibiotics with resistance-breaking features are being developed with even less compounds successfully entering the market. In fact, the Wellcome Trust's review on AMR predicts that human deaths attributable to MDR could exceed 10 million per year by 2050 with an accumulated economic cost of approximately US $\$ 100$ trillion $[2,3]$. However, already today, AMR comes along with a significant economic burden with roughly $\$ 63$ billion in costs per year in the USA alone [4].

The current resistance situation urges a speedy and conjoint effort of the scientific community, physicians and pharmacists, politicians, agriculturists and, no less important, the society. Means for reaching this goal include health promotion and disease prevention strategies, infection monitoring, improvement of hygiene standards in the clinics and in the community, together with more responsible prescription and administration of antibiotics in humans and livestock. In addition, we need to further promote the public awareness of the value of antibiotics by means of better communication policies on AMR prevalence and risks, mechanisms of transfer and spread of MDR pathogens, as well as a correct and conscious use of antibiotics. All such efforts need to be thought through and approached on a global scale. An important step toward a coordinated global response to AMR and an improved public awareness on this important topic was its discussion at the United Nations General Assembly in 2016 and 
in the meetings of the G7 and G20 groups of countries during the past 4 years. The drastic social, economic and health-related impact of the current SARS-CoV-2 coronavirus outbreak may only foreshadow the even more severe consequences that can be expected of a global AMR crisis due to an enduring run out of antibiotics. Thus, it is high time to unmistakably make clear, that "we have reached a critical point and must act now on a global scale to slow down antimicrobial resistance" [3].

\section{Current antibiotic discovery: a song of ice and fire?}

Slowing down the spread of AMR is an important task for today and the future, but it is not sufficient to avert an impending run out of antibiotics. In addition, new antimicrobial strategies (including new antibiotics, and also alternative strategies such as antivirulence agents, vaccines, etc.,) have to be explored and developed to counteract such a scenario. Here, the WHO 'Global priority list of antibiotic-resistant bacteria to guide research, discovery, and development of new antibiotics' already names the most pressing MDR strains for antibiotic discovery including Acinetobacter baumannii, Pseudomonas aeruginosa, Enterobacteriaceae ('critical priority') as well as Enterococcus faecium, Staphylococcus aureus, Helicobacter pylori, Campylobacter, Salmonella spp., Neisseria gonorrhoeae ('high priority') among others [5].

During the last decade, a number of new initiatives were started to promote antibacterial drug discovery and development. In the EU, the Innovative Medicines Initiative (IMI), a public-private partnership co-financed by the EU and the European Federation of Pharmaceutical Industries and Associations (EFPIA), supports antibiotic programs with a focus on preclinical and clinical development as well as on finding solutions to the regulatory and business challenges of new antibacterial development [6]. In the USA, the Infectious Disease Society runs the ' 10 by 20 ' program to promote the development of ten new antibiotics by the year 2020 [7]. CARB-X, a nonprofit public-private partnership dedicated to accelerating innovative antimicrobial research, was launched in 2016 by the US Department of Health and Human Services' Biomedical Advanced Research and Development Authority (BARDA) and the National Institute of Allergy and Infectious Diseases (NIAID/NIH), currently funding 18 companies from six different countries [8]. Similarly, the WHO and the Drugs for Neglected Diseases Initiative (DNDi) started the nonprofit Global Antibiotic Research and Development Partnership (GARDP) to stimulate research and development of new antibiotics and to ensure their sustainable and worldwide access [9]. In Germany, the DZIF (German Center for Infection Research), initiated and funded by the German Federal Ministry of Education and Research, runs a priority task force with a special focus on novel antibiotics with the goal to bring basic research and translational anti-infective development activities (preclinical and early clinical) back together [10]. Also, the German Research Foundation granted the Transregional Collaborative Research Center TRR261 'Antibiotic CellMAP' [11], as well as the Cluster of Excellence 'controlling microbes to fight infections' [12], two academic-driven initiatives that are intimately concerned in exploring antibiotic modes of action and how to most effectively use antibiotics to counteract pathogens in microbial communities that populate the human body. Further noteworthy, the renowned, nonprofit Gordon Research Conference organization runs a series on 'new antibacterial discovery and development' to provide a joint platform for academia, industry and government agencies to discuss and to share ideas on hit identification, target discovery and validation, chemical optimization, as well as clinical trial design and execution [13].

However, despite the fact that public and political awareness concerning AMR is rising, and a number of initiatives have been started to find new ways to counteract AMR, the efforts of big pharmaceutical companies clearly oppose this trend. This is also reflected by the fact that the number of new and innovative antimicrobial agents entering the market is declining, and looking at the active antibiotic R\&D pipelines, it is getting more than apparent that the 'golden age of antibiotic discovery' has long since passed. Even worse, the antibiotic pipeline is at risk of drying up [14]. Only 12 new antibiotics (or antibiotic combinations) have been approved since 2000 and most pharmaceutical companies have shutdown their antibiotic research programs [15]. Just recently, the pharma giants AstraZeneca (Cambridge, UK; in 2016), Novartis (Basel, Switzerland; in 2018), Sanofi (Paris, France; in 2018), and Johnson \& Johnson (NJ, USA; in 2019) stopped their active antibiotic R\&D programs, despite their joint announcement in early 2016 to further invest in antibiotic development to fight AMR ('AMR Industry Alliance'). This misguided trend is mostly due to the fact that antibiotic drugs fail to meet the criteria for satisfactory economic viability, an obvious prerequisite for the private sector in developing new antibacterial drugs. Antibiotic $\mathrm{R} \& \mathrm{D}$ programs are both laborious and challenging, novel agents have to prove superior over existing antibiotics and, once on the market, they are sparsely used due to their status as last-resort antibiotics and/or their use has to be restricted to avoid AMR. Compared with medical indications for the treatment of patients with chronic diseases 
(e.g., cancer, hypertension or diabetes therapies), antibiotic drugs provide a rather low return on investment. Obviously, in addition to public and political initiatives, we will have to reconsider the current economic rules for antibiotic R\&D to stimulate the development and production of new diagnostic tools as well as antibiotics, vaccines and other treatment alternatives to make them affordable and allow their equitable access. An alternative strategy to this current cul-de-sac may be to delink the development of diagnostics and antimicrobial therapies from price and sales volume. Then, with a new rising commercial value, for example, based on the value to society and medicine, antibiotic research may attract more of 'Big Pharma' back to the antibacterial drug discovery field.

\section{Novel antibiotics to fight resistance}

Despite the constantly declining interest of Big Pharma in antibiotic R\&D, current efforts in both academia and the private sector prove that there are still new lead compounds with excellent antimicrobial properties to find. For example, several antibacterial agents in clinical development fulfill all innovativeness criteria, defined by novel chemical class, target and mode of action as well as no cross-resistance [16].

From these, ridinilazole (previously known as SMT19969) represents a new class of bis-benzimidazole antibiotics, which is an ideal example for how the combined efforts of academic and pharmaceutical partners can successfully produce novel, potent leads. The compound arose from an academic-driven program for the design of novel bis-benzimidazoles (University of Reading and later at Queen's University Belfast), followed by further compound optimization conducted by the pharmaceutical counterpart (Summit Therapeutics, Abingdon, UK), which finally yielded the lead compound SMT19969 [17]. Extensive in vitro and in vivo data proved ridinilazole to exert promising activity and selectivity against Clostridium difficile, a common gastrointestinal pathogen, which is responsible for difficult to treat, hospital-acquired $C$. difficile infections in the elderly population [18,19]. These promising findings paved the road to ridinilazole clinical trials. Given the success of Phases I and II in healthy volunteers, an ongoing Phase III study is expected to be finalized by 2021 [20]. The molecular target of ridinilazole has yet not been elucidated; however, the antibiotic induced septation defects and filamentation of $C$. difficile cells at concentrations below the minimal inhibitory concentration (MIC), suggesting bacterial cell division as one possible target pathway [21]. Of note, bacterial cell division has emerged as a novel and promising route for antibiotic intervention in recent years, and a detailed review on this topic is available in this issue of Future Microbiology [22].

Also, brilacidin, a small arylamide agent designed from host defensins, is explored by a conjoint effort of the University of Pennsylvania and Polymedix (part of Innovation Pharmaceuticals, Inc.; MA, USA). Brilacidin (PMX30063) is a membrane-targeting antibiotic with broad-spectrum activity against clinically relevant Grampositive and Gram-negative bacteria. The bactericidal properties of brilacidin most probably derive from its membrane depolarization effects and abrogation of cell membrane functions [23]. Brilacidin has completed Phase II clinical trials for the use as an oral rinse in the treatment of oral mucositis in patients with head and neck cancer undergoing chemotherapy, and subsequent Phase III trials have been accorded with the US FDA [24]. The versatility of brilacidin allows further applications and a Phase IIb clinical trial was completed for the intravenous therapeutic use in acute bacterial skin and skin structure infections. Moreover, a Phase II trial has been planned for an enema formulation for the treatment of gastrointestinal diseases, and future clinical trials are being planned for several skin conditions and diseases [24].

Afabicin (Debio-1450 or AFN-1720, Debiopharm Group, Lausanne, Switzerland) was designed as a specific inhibitor of the staphylococcal enoyl-acyl carrier protein reductase FabI, a central enzyme acting as an elongating factor in bacterial fatty acid metabolism. Undergoing Phase II clinical trials evaluate the administration of afabicin both orally and intravenously with preliminary reports showing positive outcome for the treatment of acute bacterial skin and skin structure infections as well as bone and joint infections [25].

Nonetheless and despite reaching clinical trials, in many instances, a further development of an antimicrobial agent is discontinued due to undesired drug-driven secondary effects. This is the case for murepavadin (previously POL7080) that is developed by the biotech company Polyphor together with the University of Zurich. Murepavadin belongs to a new class of peptido-mimetic antibiotics designed from the antimicrobial peptide protegrin I. The drug targets the outer membrane of $P$. aeruginosa by binding to the lipopolysaccharide transport protein LptD, thus inhibiting its natural functions in lipopolysaccharide transport and ultimately causing cell death [26]. Polyphor developed murepavadin in two formulations, intravenous and inhaled, as a therapeutic option against carbapenem-resistant $P$. aeruginosa, which accounts for hospital-acquired and ventilator-associated bacterial pneumonia. However, Phase III clinical trials for the intravenous preparation were recently discontinued due to an increased incidence of acute kidney failure in the treated cohort (press release of 17 July 2019) [27]. 
In addition to the previous examples in Phase I-III clinical trials, there are further new and promising antibiotics with innovative mechanisms such as teixobactin, which is currently in preclinical development. Teixobactin was discovered from the unculturable Gram-negative bacterium Eleftheria terrae. This natural product is effective toward several pathogenic Gram-positive bacteria including S. aureus and Mycobacterium tuberculosis. The novelty of teixobactin lies in the concomitant sequestration of the essential cell wall precursors lipid II and lipid III, which are involved in the syntheses of peptidoglycan and teichoic acid, respectively. To date, no resistance toward teixobactin has been detected, highlighting the benefits of multiple cellular targets and also targets outside of the cell, which may reduce the effects of efflux pumps aiding AMR [28].

Ribocil is a riboswitch inhibitor that mimics flavin mononucleotide. Riboswitches consist of noncoding RNA segments within mRNA that are involved in gene regulation. Normally, ligands bind to the riboswitch aptamer and induce a conformational change, thereby facilitating the access to the transcription platform and regulating gene expression. Flavin mononucleotide riboswitches modulate the expression of genes involved in the biosynthesis and uptake of riboflavin, an essential cofactor in several enzymatic reactions. These particular riboswitches are widely conserved in pathogenic bacteria, but absent in humans. Ribocil acts as a negative regulator, which binds to the riboswitch and thereby blocks the riboflavin pathway, thus inducing bacterial cell death as a cause of riboflavin deficiency [29].

An example for a combined antimicrobial and antivirulence strategy involves the inhibition of the bacterial caseinolytic protease (Clp) by small-molecule inhibitors and/or activators. One such versatile compound class is represented by acyldepsipeptide antibiotics (ADEP) [30-34], which are reviewed in more detail in this issue of Future Microbiology [22]. Furthermore, $\beta$-lactones were explored by Böttcher and Sieber using activity-based protein profiling $[35,36]$. The inhibitory effect of $\beta$-lactones on $\mathrm{Clp}$ was reflected by a reduction of virulence factor expression in clinically relevant bacteria, for example, $\alpha$-hemolysin in $S$. aureus and listeriolysin in Listeria monocytogenes. These effects of $\beta$-lactones echoed in less severe $S$. aureus skin infections in mice and reduced $L$. monocytogenes proliferation inside macrophages [35,37]. Such groundbreaking studies encourage the use of antivirulence agents as an alternative treatment strategy. Along the lines of antibiotic intervention with bacterial protein homeostasis, the Clp system in M. tuberculosis is affected by the cyclic peptide cyclomarin A (CymA) [38]. The bactericidal effect of CymA derives from its binding to and activating the $\mathrm{AAA}+$ chaperone $\mathrm{ClpC}$, thereby stimulating $\mathrm{ClpCP}$ protease activity that leads to uncontrolled proteolysis events and finally cell death. ADEPs, $\beta$-lactones and CymA are thus promising examples that validate the deregulation of bacterial Clp protease as a novel and versatile route for antibiotic and antivirulence intervention.

Notwithstanding these promising examples, it is important to note that according to the WHO 49 antibiotics are in clinical trials, but only 15 of those are considered to be innovative [39], and thus too few new antibiotics are assumed to be approved and to enter the market within the next years. Contrariwise, AMR evolves and disseminates quickly and globally, and indeed, we should "stop referring to a coming post-antibiotic era-it's already here" [40]. More than ever, it is important to constantly foster the development of new antimicrobial strategies, with new chemical classes of antibiotics that preferably target unusual or multiple cellular structures, to counteract this alarming global trend.

\section{Acknowledgments}

The authors gratefully thank all team members of our department for their support and critical discussions.

\section{Financial \& competing interests disclosure}

The authors declare no competing interests. The authors appreciate funding by the Deutsche Forschungsgemeinschaft (DFG, German Research Foundation) - project ID 398967434 (TRR 261, TP A02) and project ID 32152271 (SFB766, TP A17) - and by the German Center for Infection Research (DZIF; TTU 09.815). The authors are further supported by infrastructural funding from the Deutsche Forschungsgemeinschaft (DFG), Cluster of Excellence EXC 2124 controlling microbes to fight infections. The authors have no other relevant affiliations or financial involvement with any organization or entity with a financial interest in or financial conflict with the subject matter or materials discussed in the manuscript apart from those disclosed.

No writing assistance was utilized in the production of this manuscript.

\section{Open access}

This work is licensed under the Attribution-NonCommercial-NoDerivatives 4.0 Unported License. To view a copy of this license, visit http://creativecommons.org/licenses/by-nc-nd/4.0/ 


\section{References}

1. World Health Organization - WHO. Global action plan on antimicrobial resistance. www.who.int/antimicrobial-resistance/publications/global-action-plan/en/

2. O'Neill J (Ed). Infection prevention control and surveillance: limiting the development and spread of drug resistance. https: //amr-review.org/sites/default/files/Health\%20infrastructure\%20and\%20surveillance\%20final\%20version_LR_NO\%20CROPS.pdf

3. O’Neill J (Ed.). Tackling drug-resistant infections globally: final report and recommendations. https://amr-review.org

4. Centers for Disease Control and Prevention. Antimicrobial resistance posing growing health threat. www.cdc.gov/media/releases/2011/p0407_antimicrobialresistance.html

5. World Health Organization. Global priority list of antibiotic-resistant bacteria to guide research, discovery, and development of new antibiotics (2017). www.who.int/medicines/publications/WHO-PPL-Short_Summary_25Feb-ET_NM_WHO.pdf?ua=1

6. Innovative Medicines Initiative. New Drugs for Bad Bugs - IMI's response to a major public health threat. https://www.imi.europa.eu/news-events/press-releases/new-drugs-bad-bugs-imis-response-major-public-health-threat

7. America IDSO. The $10 \times$ '20 Initiative: pursuing a global commitment to develop 10 new antibacterial drugs by 2020. Clin. Infect. Dis. 50(8), 1081-1083 (2010).

8. Carb-X. Combating antibiotic-resistant bacteria. www.carb-x.org

9. GARDP. Global antibiotic research and development partnership. www.gardp.org

10. DZIF - German Centre for Infection Research. TTU novel antibiotics. www.dzif.de/en/novel-antibiotics

11. Transregional Collaborative Research Center TRR261. Cellular mechanisms of antibiotic action and production (antibiotic CellMAP). https:

//uni-tuebingen.de/en/research/core-research/transregional-collaborative-research-centers-crc-trrs/crc-trr-antibiotic-cellmap/

12. Cluster of Excellence CMFI. Controlling microbes to fight infections.

https://uni-tuebingen.de/en/research/core-research/cluster-of-excellence-cmfi/

13. Gordon Research Conferences. GRC on new antibacterial discovery and

development. www.grc.org/new-antibacterial-discovery-and-development-conference/

14. Cooper MA, Shlaes D. Fix the antibiotics pipeline. Nature 472(7341), 32 (2011).

15. Nature E. Wanted: a reward for antibiotic development. Nat. Biotechnol. 36(7), 555 (2018).

16. World Health Organization - WHO. Antibacterial agents in clinical development: an analysis of the antibacterial clinical development pipeline, including tuberculosis (2017). http://apps.who.int/iris/bitstream/10665/258965/1/WHO-EMP-IAU-2017.11-eng.pdf?ua=1

17. Mann J, Taylor PW, Dorgan CR et al. The discovery of a novel antibiotic for the treatment of Clostridium difficile infections: a story of an effective academic-industrial partnership. MedChem Comm 6(8), 1420-1426 (2015).

18. Goldstein EJC, Citron DM, Tyrrell KL. Comparative in vitro activities of SMT19969, a new antimicrobial agent, against 162 strains from 35 less frequently recovered intestinal Clostridium species: implications for Clostridium difficile recurrence. Antimicrob. Agents Chemother. 58(2), 1187-1191 (2014).

19. Sattar A, Thommes P, Payne L, Warn P, Vickers RJ. SMT19969 for Clostridium difficile infection (CDI): in vivo efficacy compared with fidaxomicin and vancomycin in the hamster model of CDI. J. Antimicrob. Chemother. 70(6), 1757-1762 (2015).

20. Carlson TJ, Endres BT, Bassères E, Gonzales-Luna AJ, Garey KW. Ridinilazole for the treatment of Clostridioides difficile infection. Expert Opin. Investig. Drugs 28(4), 303-310 (2019).

21. Bassères E, Endres BT, Khaleduzzaman $\mathrm{M}$ et al. Impact on toxin production and cell morphology in Clostridium difficile by ridinilazole (SMT19969), a novel treatment for C. difficile infection. J. Antimicrob. Chemother. 71(5), 1245-1251 (2016).

22. Silber N, Matos De Opitz CL, Mayer C, Sass P. Cell division protein FtsZ: from structure and mechanism to antibiotic target. Future Microbiol. doi:https://doi.org/10.2217/fmb-2019-0348 (2020) (In Press).

23. Mensa B, Howell GL, Scott R, Degrado WF. Comparative mechanistic studies of brilacidin, daptomycin, and the antimicrobial peptide LL16. Antimicrob. Agents Chemother. 58(9), 5136-5145 (2014).

24. Innovation Pharmaceuticals. Brilacidin. www.ipharminc.com/brilacidin-1

25. Menetrey A, Janin A, Pullman J et al. Bone and joint tissue penetration of the Staphylococcus - selective antibiotic afabicin in patients undergoing elective hip replacement surgery. Antimicrob. Agents Chemother. 63(3), e01669-01618 (2019).

26. Wach A, Dembowsky K, Dale GE. Pharmacokinetics and safety of intravenous murepavadin infusion in healthy adult subjects administered single and multiple ascending doses. Antimicrob. Agents Chemother. 62(4), e02355-e02317 (2018).

27. Polyphor Ltd. Polyphor closes the Phase III PRISM studies of murepavadin intravenous formulation and evaluates further product improvement options. www.polyphor.com/news/corporate-news-details/?newsid=1800485

28. Ling LL, Schneider T, Peoples AJ et al. A new antibiotic kills pathogens without detectable resistance. Nature 517(7535), 455 (2015).

29. Howe JA, Wang H, Fischmann TO et al. Selective small-molecule inhibition of an RNA structural element. Nature 526(7575), 672 (2015). 
30. Brötz-Oesterhelt H, Sass P. Bacterial caseinolytic proteases as novel targets for antibacterial treatment. Int. J. Med. Microbiol. 304(1), 23-30 (2014).

31. Famulla K, Sass P, Malik I et al. Acyldepsipeptide antibiotics kill mycobacteria by preventing the physiological functions of the ClpP1P2 protease. Mol. Microbiol. 101(2), 194-209 (2016).

32. Pan S, Malik IT, Thomy D, Henrichfreise B, Sass P. The functional ClpXP protease of Chlamydia trachomatis requires distinct $c l p P$ genes from separate genetic loci. Sci. Rep. 9(1), 14129 (2019).

33. Malik IT, Brötz-Oesterhelt H. Conformational control of the bacterial Clp protease by natural product antibiotics. Nat. Prod. Rep. 34(7), 815-831 (2017).

34. Mayer C, Sass P, Brötz-Oesterhelt H. Consequences of dosing and timing on the antibacterial effects of ADEP antibiotics. Int. J. Med. Microbiol. 309(7), 151329 (2019).

35. Böttcher T, Sieber SA. $\beta$-lactones as specific inhibitors of ClpP attenuate the production of extracellular virulence factors of Staphylococcus aureus. J. Am. Chem. Soc. 130(44), 14400-14401 (2008).

36. Böttcher T, Sieber SA. $\beta$-lactams and $\beta$-lactones as activity-based probes in chemical biology. MedChem Comm 3(4), 408-417 (2012).

37. Böttcher T, Sieber SA. $\beta$-lactones decrease the intracellular virulence of Listeria monocytogenes in macrophages. ChemMedChem 4(8), 1260-1263 (2009).

38. Maurer M, Linder D, Franke KB et al. Toxic activation of an AAA+ protease by the antibacterial drug cyclomarin A. Cell Chem. Biol. 26(8), 1169.e1164-1179.e1164 (2019).

39. World Health Organization. Antibacterial products in clinical development for priority pathogens. www.who.int/research-observatory/monitoring/processes/antibacterial_products/en/

40. Redfield RR. US Centers for Disease Control and Prevention, CDC. U.S. Department of Health and Human Services. Antibiotic Resistance Threats in the United States, GA, USA (2019). www.cdc.gov/drugresistance/pdf/threats-report/2019-ar-threats-report-508.pdf 